\title{
Boson peak in ultrathin alumina layers investigated with neutron spectroscopy
}

\author{
D. L. Cortie,,${ }^{1,}$ M. J. Cyster, ${ }^{2}$ T. A. Ablott, ${ }^{3}$ C. Richardson, ${ }^{3}$ J. S. Smith, ${ }^{2}$ G. N. Iles $\odot,{ }^{4}$ X. L. Wang, ${ }^{1}$ D. R. G. Mitchell, ${ }^{5}$ \\ R. A. Mole, ${ }^{6}$ N. R. de Souza ${ }^{6},{ }^{6}$ D. H. Yu, ${ }^{6}$ and J. H. Cole ${ }^{2, \dagger}$ \\ ${ }^{1}$ The Institute for Superconducting and Electronic Materials, University of Wollongong, NSW 2522, Australia \\ ${ }^{2}$ Chemical and Quantum Physics, School of Science, RMIT University, Melbourne, Victoria 3000, Australia \\ ${ }^{3}$ School of Chemistry and Molecular Bioscience, University of Wollongong, NSW 2522, Australia \\ ${ }^{4}$ Space Physics, School of Science, RMIT University, Melbourne, Victoria 3000, Australia \\ ${ }^{5}$ Electron Microscopy Centre, University of Wollongong, NSW 2522, Australia \\ ${ }^{6}$ Australian Nuclear Science and Technology Organisation, Lucas Heights, NSW 2232, Australia
}

(Received 1 August 2019; accepted 30 April 2020; published 11 June 2020)

\begin{abstract}
Bulk glasses exhibit extra vibrational modes at low energies, collectively known as the boson peak. The vibrational dynamics in nanoscale alumina glasses have an impact on the performance of qubits and other superconducting devices; however, the frequency of the boson peak has not been previously measured. Here we report neutron spectroscopy experiments on $\mathrm{Al} / \mathrm{Al}_{2} \mathrm{O}_{3}$ nanoparticles consisting of spherical metallic cores with a radii from 20 to $1000 \mathrm{~nm}$ surrounded by a 3.5-nm-thick alumina glass. A low-energy peak is observed at $\omega_{\mathrm{BP}}=2.8 \pm 0.6 \mathrm{meV}$ for highly oxidized particles, indicating an excess in the density of states. The intensity of the peak scales inversely with particle size and oxide fraction, indicating a surface origin, and is redshifted by $3 \mathrm{meV}$ with respect to the van Hove singularity of $\gamma$-phase $\mathrm{Al}_{2} \mathrm{O}_{3}$ nanocrystals. Molecular-dynamics simulations of $\alpha-\mathrm{Al}_{2} \mathrm{O}_{3}, \gamma-\mathrm{Al}_{2} \mathrm{O}_{3}$ and $\alpha-\mathrm{Al}_{2} \mathrm{O}_{3}$ show that the observed boson peak is a signature of the ultrathin glass surface and the characteristic frequency is reduced compared to the peak in the bulk glass.
\end{abstract}

DOI: 10.1103/PhysRevResearch.2.023320

\section{INTRODUCTION}

Amorphous materials are common in nature and their associated glass transitions have far ranging consequences from geology to nanoelectronics [1-3]. Despite the structural differences between oxide, metal, and polymer glasses, there are striking universal features in their dynamic properties [4]. These include an enhancement in the vibrational density of states (DOS) at terahertz (THz) frequencies and a corresponding plateau in the heat conductivity at low temperatures [4]. This manifests as an enigmatic feature in Raman and neutron spectroscopy observable in the $\mathrm{THz}(\mathrm{meV})$ frequency range called the "boson peak" (BP) [2,5]. One characteristic length scale is defined by the ratio of the speed of sound $(v)$ to the $\mathrm{BP}$ frequency $v_{\mathrm{BP}}$ and falls in the range $l_{c} \approx 0.3-5.0 \mathrm{~nm}$ for most glasses [6]. It is interesting to consider the situation for nanoscopic glasses with dimensions approaching $l_{c}$ since this can provide clues into the nature of the excitations responsible for the BP, and the effects of spatial inhomogeneity within the glass. Only a handful of experiments have explored this question. For example, when polymer glasses are confined in spherical nanopores, an asymmetric BP is observable for

\footnotetext{
*dcortie@uow.edu.au

†jared.cole@rmit.edu.au

Published by the American Physical Society under the terms of the Creative Commons Attribution 4.0 International license. Further distribution of this work must maintain attribution to the author(s) and the published article's title, journal citation, and DOI.
}

smaller pore dimensions, and $v_{\mathrm{BP}}$ can be up-shifted or downshifted depending on whether hard or soft boundary conditions occur [7,8]. For supercooled liquid water in nanopores, a BP can also be observed for some special conditions $[9,10]$. In oxide glasses, however, the existence of the BP in nanostructures has received less attention. Such materials are widely used as ultra-thin-film layers (1-3 nm thick) in superconducting junctions and complementary-metal-oxide-semiconductor devices. It is expected that the confinement effect on the BP for ultrathin oxide films will be qualitatively different from spherical confinement in a pore. To the best of our knowledge this situation has not been explored to date in any experiments or calculations. One key difference is that a thin-film glass has a higher surface area than a glass droplet in a pore, and may also experience anisotropic vacuum or substrate interactions that modify the boundary conditions. On general grounds, interfaces are expected to create a strong source of inhomogeneity at a spatial scale comparable to $l_{c}$ which could shift or broaden the BP considerably. To date, a well-defined BP has been observed at the surface of a $\mathrm{SiO}_{2}$ semi-infinite crystal $[11,12]$, however there appears to be no similar information available for ultrathin oxide glasses. In particular, the features of the $\mathrm{BP}$ remain unknown for the important class of alumina $\left(\mathrm{Al}_{2} \mathrm{O}_{3}\right)$ glasses.

Alumina glasses are far less studied than the well-known bulk oxide glasses. Unlike the canonical glasses (e.g., $\mathrm{SiO}_{2}$ ), alumina is not an intrinsic glass former under most circumstances [13]. However, like the majority of oxides, alumina can exist as amorphous phases in metastable thin films, and the surface/interface energy can act to stabilize glassy phases at the expense of the crystalline phases $[14,15]$. The latter is 
the case for alumina $\mathrm{Al}_{2} \mathrm{O}_{3}$ glass since this is generally only stable as thin surface layers [15], as small nanoparticles [16], or in high surface area mesoporous structures [17]. Amorphous $\mathrm{Al}_{2} \mathrm{O}_{3-x}$ layers (usually notated as $\mathrm{AlO}_{x}$ ) are widely used as junctions in electronics and superconducting devices. The lack of a bulk alumina glass means that, to the best of our knowledge, the frequency and very existence of the $\mathrm{BP}$ has not been measured in any form of glassy alumina. This basic question has taken on an applied dimension with the development of superconducting electronics based on thin $\mathrm{Al} / \mathrm{AlO}_{x} / \mathrm{Al}$ junctions. The stoichiometry in such oxides can vary yet they often approach the stoichiometry and structure of the native oxide $\left(\mathrm{Al}_{2} \mathrm{O}_{3}\right)$ [18]. The self-limiting nature of the oxidation of aluminium provides an ideal fabrication method for producing uniform, nanoscale insulating layers which are the key to superconducting quantum interference devices [19] and superconducting qubits [20-23]. Yet a significant drawback of this material is that two-level systems (TLSs) dominate the low-temperature physics of the alumina glass below $1 \mathrm{~K}$ [24] and limit the coherence, fidelity, and reproducibility of superconducting devices [3,25]. One candidate for the origin of the TLS is the ionic motion of atoms, or groups of atoms, between two configurations in a double potential well [3]. The realization of this hypothesis relies on quantum tunneling at low temperature at $\mathrm{GHz}$ frequencies. It is reasonable to expect that analogous hops may occur through the same structural motifs at higher temperature and at higher frequencies (THz), once the thermal energy is sufficient to overcome the meV-scale energy barrier to allow classical motion [26]. It is therefore essential to understand the microscopic atomic dynamics in nanoscale alumina at $\mathrm{THz}$ frequencies. To measure the picosecond dynamics in ultrathin alumina, we performed inelastic neutron spectroscopy on well-characterized $\mathrm{Al} / \mathrm{Al}_{2} \mathrm{O}_{3}$ structures (Fig. 1). Neutron spectroscopy is the ideal tool to study atomistic dynamics in complex structures because it offers both energy and (reciprocal) spatial resolution. Time-of-flight spectroscopy is well suited for studying the $\mathrm{meV}$-scale $(\mathrm{THz})$ spectra of polycrystalline nanopowders [27-29] and glasses [30].

\section{METHODS}

\section{A. Experimental}

Spherical aluminium nanoparticles were supplied by U.S. Research Nanomaterials, where they were produced by a high-temperature electrical explosive method [31]. The particles were deliberately exposed to ambient atmosphere to spontaneously form ultrathin amorphous oxide skins which are well known to passivate aluminium surfaces. Neutron spectroscopy was performed using the cold neutron time-offlight spectrometer (PELICAN) [32] at the Australian Nuclear Science and Technology Organisation operating at incident wavelengths of 4.690 and $2.345 \AA$. The samples were mounted in a vanadium can, and the empty can signal was subtracted at each temperature. The detector efficiency in the data was normalized using a vanadium standard. As moderate heat treatments are known to lead to crystallization of $\mathrm{Al}_{2} \mathrm{O}_{3}$ the samples were initially studied in the as-exposed state. In the Appendix, additional data are presented for particles heat (a)

(b)
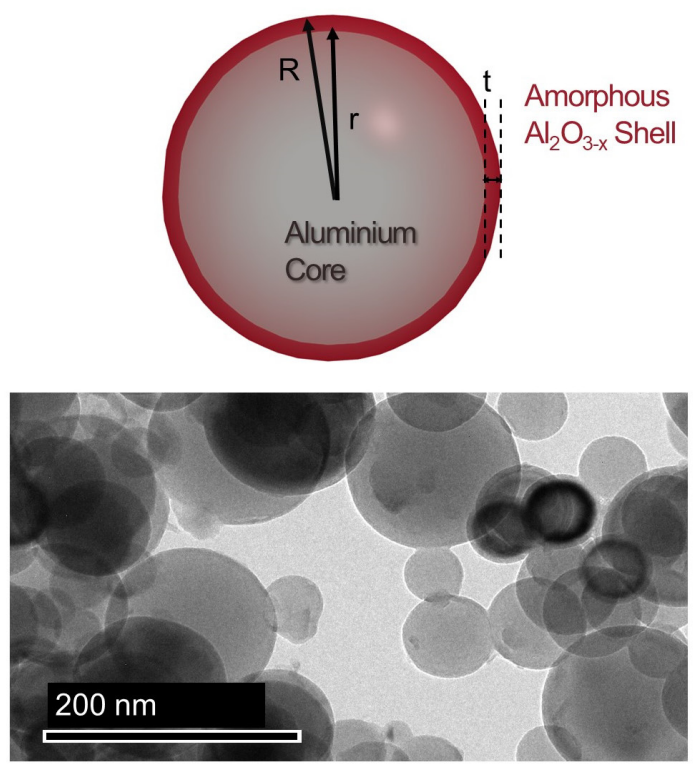

(c)

(d)
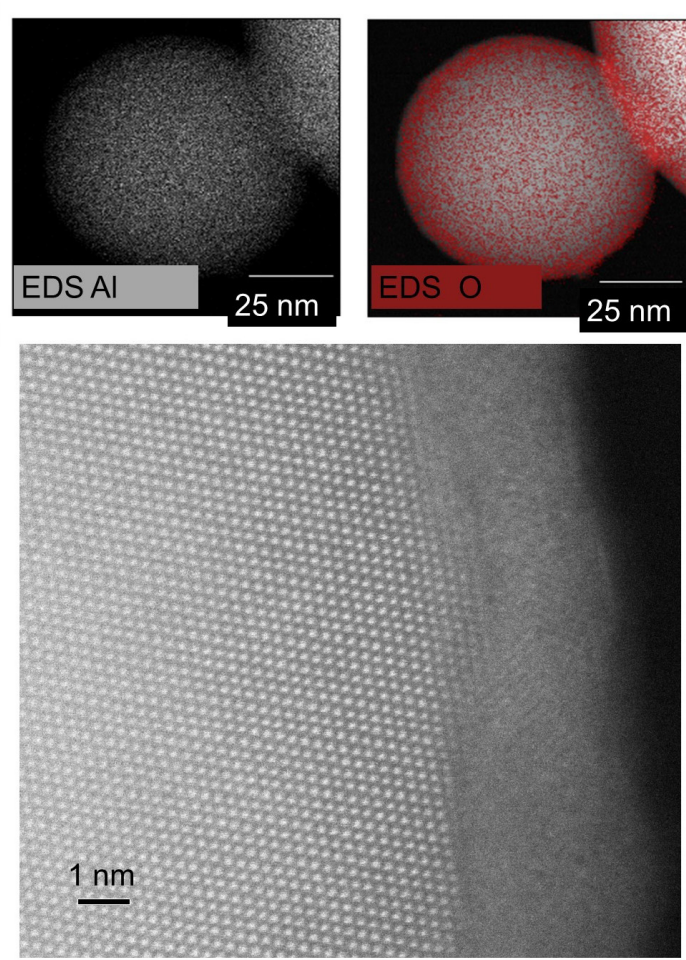

FIG. 1. (a) Schematic illustration of the core-shell structure formed by the native oxide on aluminium nanoparticles described by an outer radius $R$, an inner radius $r$, and a thickness $t$. (b) Low magnification TEM image of the ensemble of spherical $\mathrm{Al} / \mathrm{Al}_{2} \mathrm{O}_{3}$ particles with $\langle R\rangle=35 \mathrm{~nm}$. (c) Energy-dispersive $\mathrm{x}$-ray spectroscopy (EDS) maps of the aluminium profile in spherical particles and oxygen profile surrounding the particles. (d) HAADF scanning TEM image of the interface between the crystalline core and oxide shell.

treated in situ at $450 \mathrm{~K}$. To study the nanostructure morphology, some of the powders were dispersed on carbon grids, and scanning transmission electron microscopy was performed using a JEOL-ARM200F operating at $200 \mathrm{kV}$ which is equipped with aberration correction and a high-angle angular dark field (HAADF) detector. Additional low-magnification TEM 
images were captured using a JEOL-JEM 2010 for hundreds of particles to obtain a statistical distribution of the radii. $\mathrm{X}$-ray diffraction was conducted on a PANalytical Empyrean XRD using $\mathrm{Cu}-\mathrm{K} \alpha$ radiation $(\lambda=1.5406 \AA)$ and the excess peak broadening in the nanoparticles was calculated by reference to a bulk crystalline $\mathrm{Al}_{2} \mathrm{O}_{3}$ resolution standard. Simultaneous thermogravimetric-differential scanning calorimetry was performed using a NETZSCH STA449F3 Jupiter. The samples were placed in an aluminium pan and heated at 10 $\mathrm{K} / \mathrm{min}$ under $\mathrm{N}_{2}$ flow at $20 \mathrm{~mL} / \mathrm{min}$.

\section{B. Theoretical}

Molecular dynamics (MD) simulations were performed using the General Utility Lattice Program [33]. Calculations of the atomic trajectories were conducted for crystalline $\alpha$ and $\gamma$ phases, and large amorphous- $\mathrm{AlO}_{x}$ cells. All calculations used periodic boundary conditions. Models of $\mathrm{Al} / \mathrm{AlO}_{x}$ surfaces were also studied by constructing metal/metal-oxide bilayers and including a vacuum boundary in one direction. The force fields between atoms were modeled via the StreitzMintmire empirical potential [34] which includes an electronegativity correction [35]. To produce amorphous oxides with a reduced density of 0.8 times the density of crystalline $\mathrm{Al}_{2} \mathrm{O}_{3}$, in accordance with previous computational $[36,37]$ and experimental [38] works, cubes were cut from the $\alpha$ $\mathrm{Al}_{2} \mathrm{O}_{3}$ crystal with a side length of $16 \AA$. The cell parameters and atomic coordinates were then scaled to the final size of 20 $\AA$ cubed. These structures were then simulated at $4300 \mathrm{~K}$ for 10 ps to create disorder before the temperature was linearly reduced to $300 \mathrm{~K}$ over a further 10 ps to quench the structure in an amorphous configuration. Surfaces were prepared by adding an aluminium crystal layer to the simulation cell and performing a geometry optimization to reconstruct the $\mathrm{Al} / \mathrm{AlO}_{x}$ interface. The resulting structures were simulated for a total of $50 \mathrm{ps}$ at $100 \mathrm{~K}$ where the equations of motion were solved using a time step of $d t=1 \mathrm{fs}$. The intermediate neutron scattering function was calculated from the spatial coordinates of the MD trajectories, and Fourier transformed to get the simulated coherent scattering function $S(\omega, q)$ using the NMoldyn software [39]. This was weighted by the coherent neutron scattering cross sections for $\mathrm{Al}$ and $\mathrm{O}$, which is the dominant contribution. To obtain $S(\omega)$, the resulting $S(\omega, q)$ was integrated over the same $E / q$ window as accessible in experiment. The observable window is determined by the energy and momentum conservation constraints on the neutron:

$$
\frac{q^{2} \hbar^{2}}{2 m_{n}}=2 E_{i}-\hbar \omega-2 \sqrt{E_{i}\left(E_{i}-\hbar \omega\right)} \cos (2 \theta)
$$

where $m_{n}$ is the rest mass of the neutron, $E_{i}$ is the kinetic energy of the incident neutron, $\hbar \omega$ is the energy transfer during the neutron scattering event, and $\theta$ is the scattering angle limit set by the detector coverage. To model the PELICAN data, we adopted $10^{\circ}<2 \theta<110^{\circ}$ [32] and the same incidence energies as in the experiment. The MD only simulate the thermally populated classical dynamics, which result primarily in energy-gain processes for the neutron (characterized by a negative energy-transfer $\hbar \omega$ ). However, neutron spectroscopy is also sensitive to neutron-energy-loss processes where the neutron creates the excitation. In order to simulate the effect of thermal population on the asymmetry in neutron energy loss or energy gain processes, we multiplied the simulated $S(\omega, q)$ by the Bose-Einstein factor to give the classical approximation to the detailed balance factor in the spectra, such that

$$
S(-\omega, q)=e^{-\hbar \omega /\left(2 k_{B} T\right)} S(\omega, q) .
$$

\section{RESULTS AND DISCUSSION}

The ambient exposure of the Al particles results in coreshell nanoparticles [Fig. 1(a)] where $t$ is the thickness of the oxide skin, and $R$ is the total radius. Past studies reported that the surface oxide has a thickness between 2 and $4 \mathrm{~nm}$ which varies slightly for different aluminium facets $[14,40]$. This was directly confirmed in the spherical particles using transmission electron microscopy for the exposed Al samples [Figs. 1(b)-1(d)], indicating the oxide shell has $t=3.5 \pm 0.5$ nm with excellent uniformity across the different particles. Three nanopowder samples were measured, each consisting of an ensemble of spheres [e.g., Fig. 1(b)] characterized by different average radii of $\langle R\rangle=35 \pm 18,45 \pm 20$, and $350 \pm 55 \mathrm{~nm}$, where the error bar is the standard deviation. Atomic resolution imaging shows that the aluminium core is crystalline, whereas the external region is amorphous, as can be seen in the HAADF image in Fig. 1(d). Figure 2 shows the x-ray diffraction for the various samples. The only Bragg reflections that can be detected are those of crystalline Al from the core regions, indicating the thin oxide layer is amorphous. The inset highlights the peak broadening caused by the finite size of the particles. Fitting to the Scherrer formula is challenging because the finite-size broadening is very subtle and near the instrument resolution, however this is consistent with the particle dimensions obtained from the TEM. A comparison of the largest and smallest samples is shown in the superposition of two TEM images [Fig. 2(b)]. Energy-dispersive x-ray spectroscopy mapping of the largest samples shows that the oxide skin is the same thickness as in the small samples [Fig. 2(c)]. This indicates that, while there is significant polydispersity in the aluminium core sizes, the thickness of the oxide shell layers is monodisperse.

By using neutron spectroscopy to study the fine core-shell oxidized Al particles, we detected an inelastic excitation in the $q$-integrated neutron scattering intensity $S(\omega)$ [as shown in Fig. 3(a)] which is not found in aluminium or crystalline alumina. This is attributed to the oxide shell which constitutes a large fraction of the overall signal. According to simple geometric arguments, the volume fraction of the oxide shell is given by $V_{F}=\left(t^{3}+3 R^{2} t-3 R t^{2}\right) / R^{3}$, and the mass fraction of the oxide is increased by the higher mass density of the oxide. Thus, in smaller particles where $R \approx 20-60 \mathrm{~nm}$, a substantial fraction of the overall mass is in the oxide skin (20-70\%). The coherent neutron scattering cross section for oxygen (with a scattering length of $b_{\mathrm{O}}=5.803 \mathrm{fm}$ ) is also nearly twice that of aluminium $\left(b_{\mathrm{Al}}=3.45 \mathrm{fm}\right)$, thus the oxide signal is estimated to be enhanced by a further factor of $\frac{\rho_{\mathrm{AlO}}\left(b_{\mathrm{O}}+b_{\mathrm{Al}}\right)}{\rho_{\mathrm{AlO}}\left(b_{\mathrm{O}}\right)} \frac{M_{\mathrm{Al}}}{M_{\mathrm{AlO}}} \approx 2$ where $\rho$ is the mass density and $M$ is the molar mass. The observed inelastic neutron spectroscopy (INS) feature attributed to the alumina glass is very similar to 
(a)

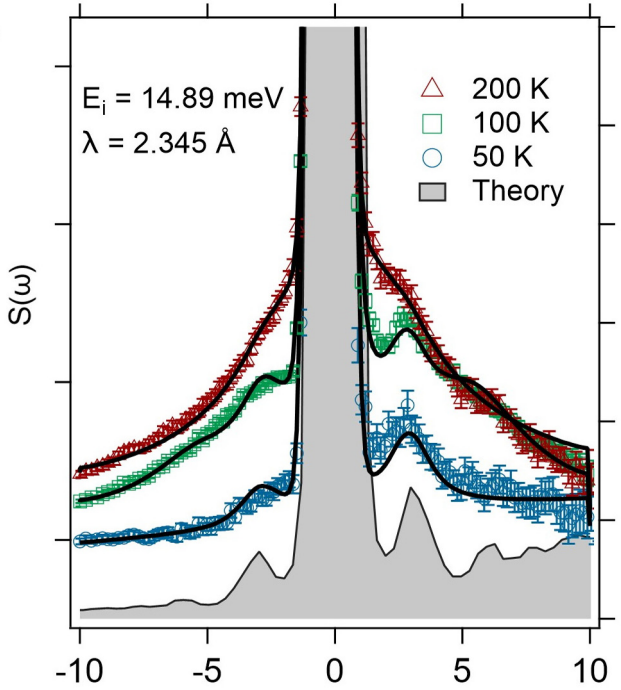

(b)

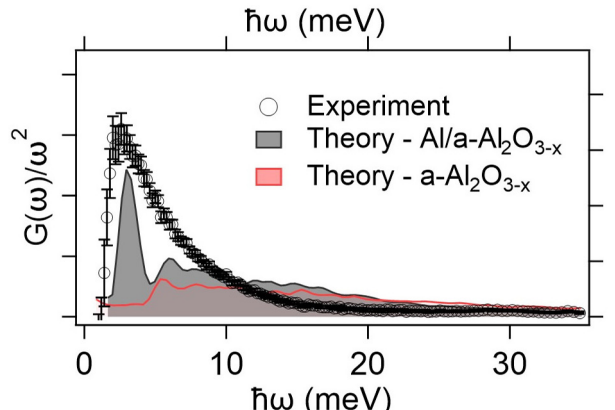

(c)

(d)
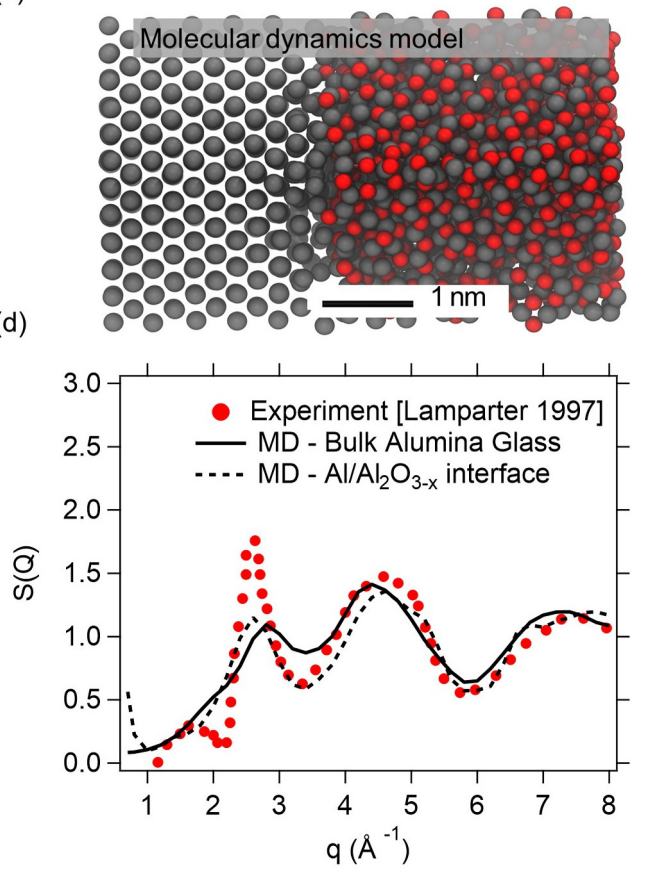

FIG. 2. (a) X-ray-diffraction patterns for the various samples show the Bragg peaks at the positions of the metallic Al reference. The inset shows an enlarged region near the (111) Al Bragg reflection, indicating the subtle Scherrer broadening from finite size. (b) TEM image for the $\langle R\rangle=35 \mathrm{~nm} \mathrm{Al}$ nanoparticles (red) superimposed on an image of the $\langle R\rangle=350 \mathrm{~nm}$ particles (gray). (c) Energy-dispersive $\mathrm{x}$-ray spectroscopy map showing the oxide thickness of $3.5 \mathrm{~nm}$ on a large particle from the $\langle R\rangle=350 \mathrm{~nm}$ ensemble (left). (a)

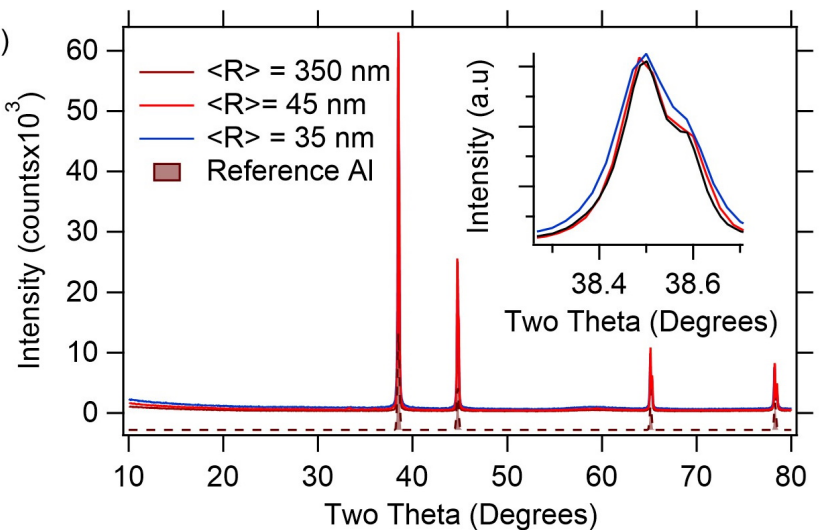

(b)
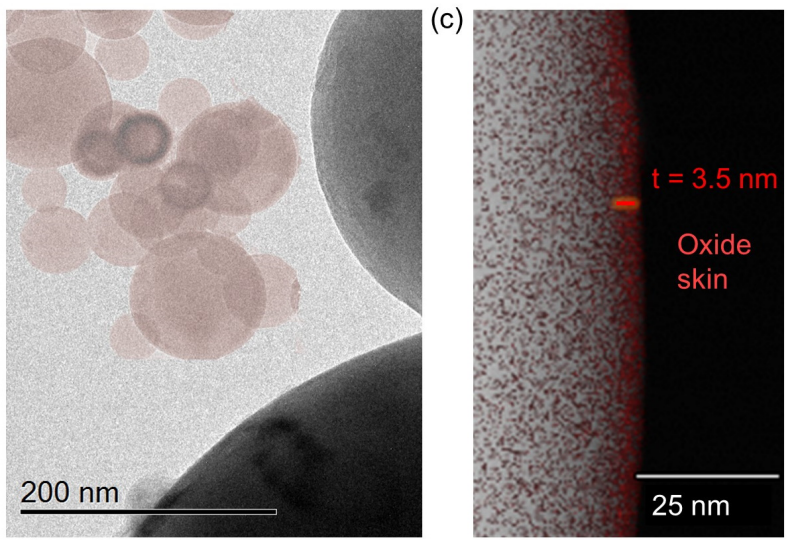

FIG. 3. (a) Inelastic neutron scattering intensity $S(\omega)$ at several temperatures. The solid lines are fits including the detailed balance factor. The theoretical spectrum calculated from the MD calculations is offset below for clarity. (b) Generalized DOS measured by inelastic neutron spectroscopy for $\langle R\rangle=35 \mathrm{~nm}$ particles at $100 \mathrm{~K}$ scaled by $w^{-2}$. The shaded regions are those calculated from the $\mathrm{MD}$ of an $\mathrm{Al} / \mathrm{Al}_{2} \mathrm{O}_{3}$ interface and a hypothetical bulk $\mathrm{Al}_{2} \mathrm{O}_{3}$. (c) Snapshot of a MD configuration of the 3-nm-thick glass interface on a crystalline substrate. (d) Calculated neutron elastic structure factor from the MD models compared with previously published experimental data.

the spectral signature of the $\mathrm{BP}$ in bulk amorphous $\mathrm{SiO}_{2}$ [30], however the frequency is reduced by $40 \%$. By using neutrons with an incident energy of $14.89 \mathrm{meV}$, the feature can be resolved in both the neutron energy-loss and energy-gain modes, thereby allowing for observation of the temperaturedependent asymmetry. As shown by the solid black lines in Fig. 3(a), the spectra can be fitted using a combination of two Lorentzians and a Gaussian, weighted by the thermal balance factor. The latter indicates the spectrum obeys Bose-Einstein statistics, a key signature of a bosonic excitation common to all glass BPs. Figure 3(b) shows the generalized DOS measured in the neutron spectroscopy in reduced units scaled by $\omega^{-2}$. This exhibits an excess of modes centered on $\omega_{\mathrm{BP}}=$ $2.8 \pm 0.6 \mathrm{meV}$, as found for BPs in glasses, and van Hove singularities $(\mathrm{vHs})$ in crystals.

Precise comparisons can be made between the INS experiments and the MD calculations using a well-established framework discussed in Ref. [39]. An example of the crystal-glass $\mathrm{Al} / \mathrm{Al}_{2} \mathrm{O}_{3}$ surface model is shown in Fig. 3(c). 
Figure 3(d) compares the glass elastic structure factor in the simulations with past experimental data [41] showing good agreement for both the pure $\mathrm{Al}_{2} \mathrm{O}_{3}$ and $\mathrm{Al} / \mathrm{Al}_{2} \mathrm{O}_{3}$ simulations. The shaded area in Fig. 3 compares the calculated inelastic neutron spectra for a 3-nm oxide skin interface on an aluminium substrate at $100 \mathrm{~K}$, where the main experimental features are well captured by the model (the theory curve is offset for clarity). The vibrational DOS $G(\omega)$ calculated from the velocity autocorrelation functions, normalized to Debye units scaled by $\omega^{-2}$, is shown in Fig. 3(b). While both pure amorphous $\mathrm{Al}_{2} \mathrm{O}_{3}$ and $\mathrm{Al} / \mathrm{Al}_{2} \mathrm{O}_{3} \mathrm{MD}$ simulations predict a $\mathrm{BP}$ between 0 and $20 \mathrm{meV}$, the models predict a sharp BP in the ultrathin oxides with a lower frequency $\left(\omega_{\mathrm{BP}}=3 \mathrm{meV}\right)$. In contrast, a broad $\mathrm{BP}$ is predicted in the hypothetical bulk glass $\left(\omega_{\mathrm{BP}}=10-20 \mathrm{meV}\right)$. The calculations for thin $\mathrm{Al} / \mathrm{Al}_{2} \mathrm{O}_{3}$ layer match well with the present experiment. While our bulk calculations are broadly in agreement with past $a b$ initio MD calculations [42], there is no direct experiment available for bulk amorphous alumina.

To assess the dispersion of the excitation in the nanoscale alumina, Fig. 4(a) shows the experimental $S(\omega, q)$ powderaveraged neutron scattering map for small $(\langle R\rangle=35 \mathrm{~nm})$ particles at $100 \mathrm{~K}$ in terms of the reciprocal space vector magnitude $(q)$ and the energy transfer. In the section on the right of the figure, the diffraction pattern is plotted where the latter was extracted from the zero-energy (elastic) slice of the data. Although the nanoglass is easily concealed in the diffraction measurements, which are dominated by the crystalline aluminium peaks, it shows up as a strong feature in the inelastic neutron spectroscopy with a peak at $\omega_{\mathrm{BP}}=2.8$ $\pm 0.6 \mathrm{meV}$. The energy of the low-energy feature $(2.8 \mathrm{meV})$ is independent of $q$ as shown by the dashed line in the figure, although the intensity does vary with $q$. The absence of dispersion indicates a quasilocalized mode. The intensity is present over a broad streak of $q$, as expected for a glass feature, and not sharply modulated at the metal's Bragg points, indicating it originates from a different spatial scale. Indeed, the scattering intensity increases from 3 to $4 \AA^{-1}$, which is the characteristic $\mathrm{Al}-\mathrm{O}$ distance in the glass tetrahedra. In common with the $\mathrm{BP}$ in $\mathrm{SiO}_{2}$, the inelastic signal peaks at the second elastic maxima in the glass static structure factor because the first maximum in $S(q)$ corresponds to length scales of connected tetrahedra that are less rigid than one tetrahedron, leading to a less pronounced peak [2]. For the largest particles $(\langle R\rangle=350 \mathrm{~nm})$ the feature is only barely detectable when plotted on the same scale and normalized by mass [Fig. 4(b)], yet the aluminium diffraction peaks are sharper and stronger. This is consistent with the tiny oxide fraction $(<1 \%)$ in the bigger particles, which is expected as the oxide skin has almost the same uniform thickness, whereas the aluminium cores are very different sizes [Figs. 2(c) and 2(d)]. Theoretically, the thickness of the skin is determined by the thermodynamic stability criteria of the oxide which depends on surface energy and tends to disfavor growth beyond 3-4 $\mathrm{nm}[15,16]$. The different shell-core ratios explain why the intensity of the observed feature scales using the volumefraction and neutron enhancement factors discussed earlier. We note that the $\omega_{\mathrm{BP}}$ for the peaks in all of the samples are similar within $\approx 1 \mathrm{meV}$ of each other, which is essentially
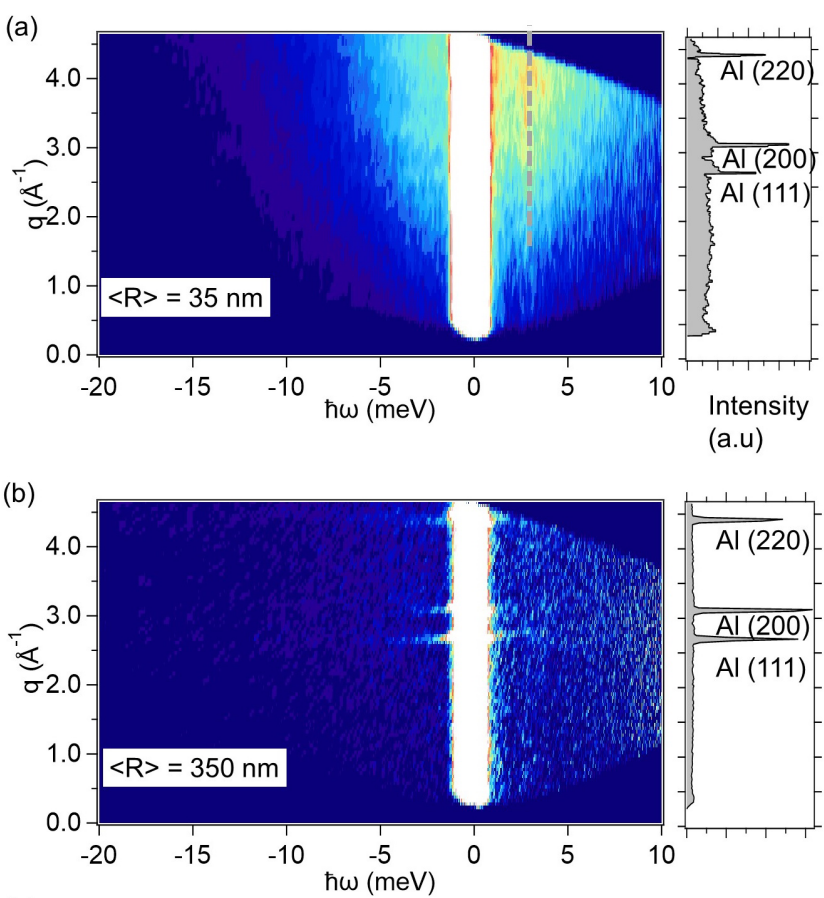

(c)

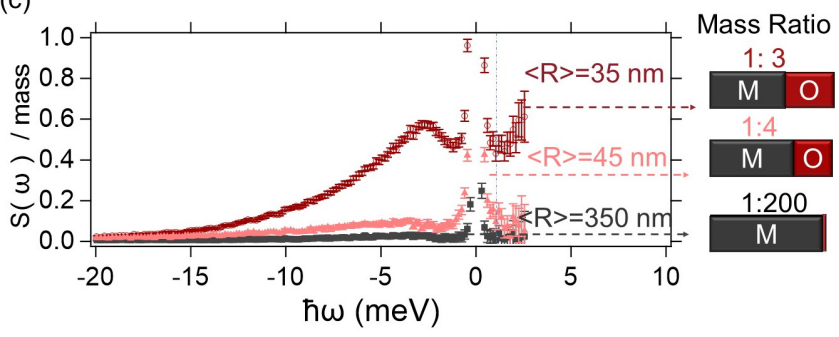

FIG. 4. (a) $S(\omega, q)$ map for the $\langle R\rangle=35 \mathrm{~nm} \mathrm{Al} \mathrm{nanopowder}$ showing the $q$ dependency of the excitation with the diffraction pattern (right) extracted from the elastic channel. (b) $S(\omega, q)$ map for $\langle R\rangle=350 \mathrm{~nm}$ with the $(E=0)$ diffraction pattern (right). (c) $q$-integrated $S(\omega)$ normalized by sample mass for the different $\mathrm{Al} / \mathrm{Al}_{2} \mathrm{O}_{3}$ nanopowders.

within uncertainty determined by experiment resolution and fitting procedure. Generally it is believed that the substratestrain interactions, or curvature, can introduce effective pressure in crystalline particles [43], which can shift vibrational frequencies, however the effect is not easy to detect in the nanoscale alumina glass.

Although the data for nanoalumina display striking similarities to $\mathrm{SiO}_{2}$ bulk glasses, it would be remiss if we failed to note that other spectroscopic studies of nanopowders have reported anomalous BP features. These have been observed even in materials such as nanocrystalline $\mathrm{Fe}[44]$ and $\mathrm{TiO}_{2}$ [28] where no such feature would be expected. Those studies did not attribute this to an interfacial glass; however, it is clear that future work needs to carefully check for the presence of amorphous skins since these potentially form on many nanoparticles, particularly metal ones. One interesting alternate proposal was that weak coupling between nanocrystallites in a nondispersed powder leads to a "microstructural" BP analogous to disordered solids [45]. To explore this, we performed control measurements on other nanocrystalline 

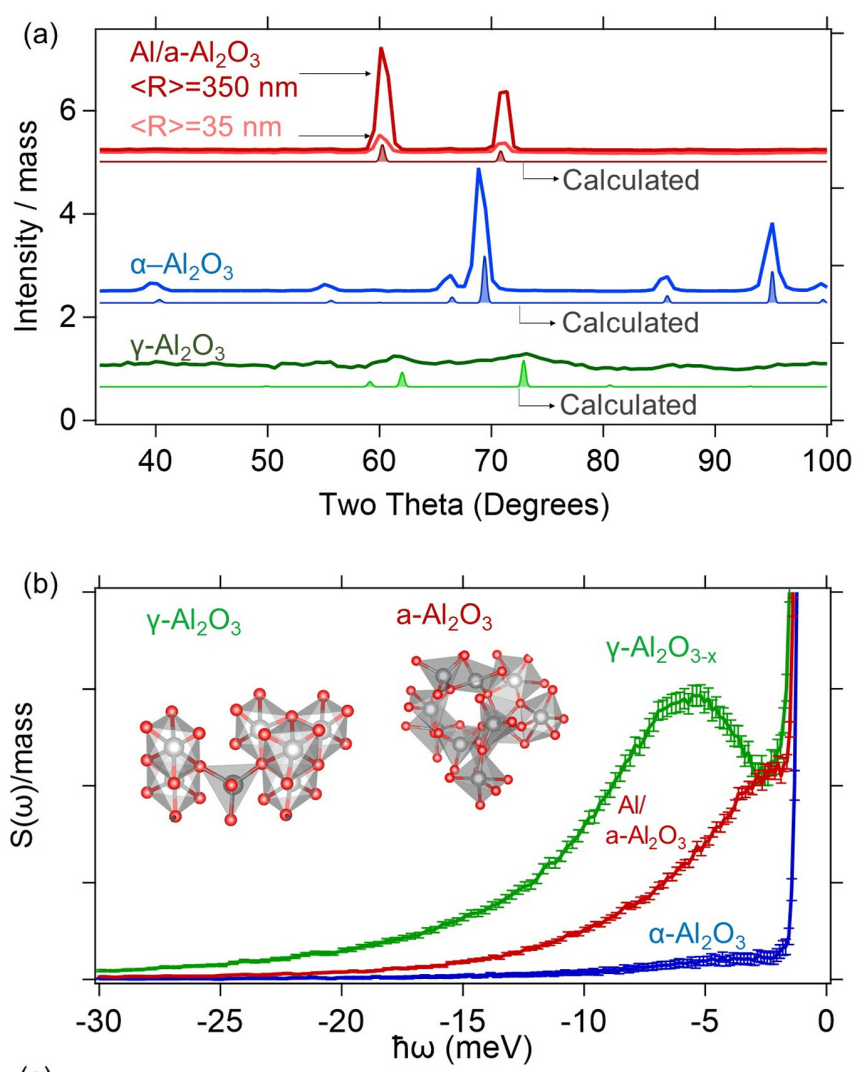

(c)

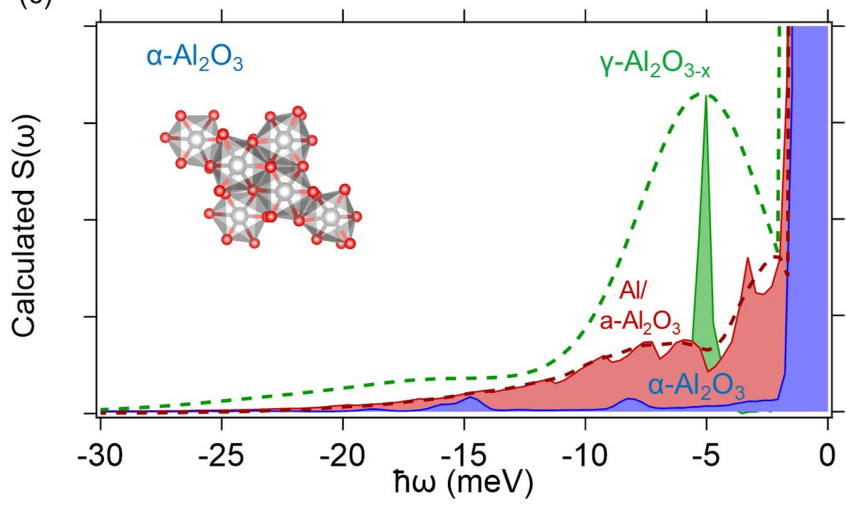

FIG. 5. (a) Neutron-diffraction patterns at $\lambda=2.345 \AA$ for $\mathrm{Al} / \mathrm{Al}_{2} \mathrm{O}_{3}, \gamma-\mathrm{Al}_{2} \mathrm{O}_{3}$, and $\alpha-\mathrm{Al}_{2} \mathrm{O}_{3}$ taken from the elastic channel. (b) $q$-integrated inelastic neutron scattering intensity for $\gamma-\mathrm{Al}_{2} \mathrm{O}_{3}$ and $\alpha-\mathrm{Al}_{2} \mathrm{O}_{3}$ nanopowders compared with the $\mathrm{Al} / \mathrm{Al}_{2} \mathrm{O}_{3}$ core-shell particles, normalized by sample mass. (c) Simulated $S(\omega)$ using classical force-field MD simulations for infinite periodic cells of $\gamma$ $\mathrm{Al}_{2} \mathrm{O}_{3}$ and $\alpha-\mathrm{Al}_{2} \mathrm{O}_{3}$ and the $\mathrm{Al} / \mathrm{Al}_{2} \mathrm{O}_{3}$ interface. The shaded regions assume the instrument resolution of $1 \mathrm{meV}$, whereas the dashed lines indicate the effect of including broadening in the simulations from finite size or disorder. The insets are snapshots from small sections of the MD models showing the different tetrahedral and octahedral networks.

aluminium oxides all of which had a similar, low packing density $(25 \%)$.

All of the nanocrystalline alumina oxides have distinctive elastic features and inelastic phonon spectra related to their intrinsic atomic structural differences. Figure 5(a) displays the elastic-channel neutron-diffraction patterns for the dif- ferent nanosized aluminium and aluminium oxide samples, along with the calculated patterns from the Inorganic Crystal Structure Database. The corundum-type and aluminium cores are of good crystal quality, evident in the strong Bragg peaks, whereas the $\gamma$ particles are only barely crystalline. Figure 5(b) compares the inelastic neutron spectroscopy for various samples all measured under identical conditions at $100 \mathrm{~K}$. It is clear that the spectra are very different despite having similar packing densities. The corundum phase shows almost no features below $10 \mathrm{meV}$, whereas both the $\gamma$ and $\mathrm{Al} / \mathrm{Al}_{2} \mathrm{O}_{3}$ particles have strong low-energy features. The difference between the samples excludes the possibility of some "universal microstructural" BP. Furthermore, the agreement with theoretical calculations strengthens the argument that the neutrons probe the intrinsic atomistic dynamics, and nanoparticle-nanoparticle interactions are of secondary, or negligible, importance. Figure 5(c) shows the theoretical neutron spectra calculated from the MD for periodic boundary conditions which do not include internanoparticle interactions. For example, in agreement with the MD theory, the first vHs in pure $\gamma$ oxide has its first vHs at $7 \mathrm{meV}$. The latter is also in agreement with a previously published $a b$ initio lattice dynamics [46]. Meanwhile, also in agreement with experiment, there are very few features observable for corundum because the first strong vHS occurs at much higher energy $(30 \mathrm{meV})$ [47], and thus is thermally depopulated at the measurement temperature of $100 \mathrm{~K}$.

As in bulk forms, the intrinsic differences between the crystalline and amorphous alumina polymorphs reflect the characteristics of the different polyhedra networks which determine the degrees of freedom and constraints on the rigidunit motions. The insets of Fig. 5(b) are snapshots of the tetrahedra and octahedra in the MD simulations of the various phases. The $\gamma$ phase has more in common with the amorphous phase in that it has similar bond lengths and features a large fraction of tetrahedral units $(40 \%)$ [46]. Unlike the octahedral units in corundum, corner-sharing tetrahedra have few constraints on their motion because of the lower number of vertices. This in turns offers additional low-energy modes of distortion, some of which are termed "floppy modes." The alumina glass has an average Al-O coordination number of 4.5 with a larger fraction of corner-sharing tetrahedra. Therefore, the BP in glassy alumina, which is redshifted with respect to the vHs of the crystalline $\gamma$ phase, has close analogies with the BP in amorphous silica, which is redshifted with respect to the vHS of crystalline crystobalite [48].

While the primary frequencies match between MD theory and experiment, the experiment shows considerable broadening attributed to finite-size effects that confine the traveling motion of the phononic excitations. These are real features because the broadening exceeds $1 \mathrm{meV}$, whereas the instrument resolution is $0.14-0.6 \mathrm{meV}$ [32]. It has been proposed that interfacial scattering reduces phonon (and magnon) lifetimes in nanocrystals leading to line broadening of the order $\Gamma=$ $h \frac{2 v}{L}$ where $v$ is the speed of sound and $L$ is the size of the particle/domain $[27,49]$. Assuming the speed of sound to be $9 \mathrm{~km} / \mathrm{s}$ [50], $\Gamma$ is $1 \mathrm{meV}$ for a crystallite with $L=70 \mathrm{~nm}$. Experimentally, the broadening is closer to $1.5 \mathrm{meV}$ in the aluminium particles and $6 \mathrm{meV}$ in the $\gamma-\mathrm{Al}_{2} \mathrm{O}_{3}$ consistent with the smaller particle sizes in the latter. The dashed lines in 
Fig. 5(c) show the effect of including linewidth broadening for the simulated spectra. As the $\mathrm{BP}$ for the $\mathrm{Al} / \mathrm{Al}_{2} \mathrm{O}_{3}$ particles has a low dispersion, $v \approx 0 \mathrm{~km} / \mathrm{s}$, it does not appear to be broadened to the same degree as the ordinary phonon features. This is consistent with a quasilocalized excitation which, unlike traveling phonon plane waves, does not experience strong boundary scattering.

\section{CONCLUSION}

The experimental and theoretical data provide compelling evidence for intrinsic low-energy vibrational modes in ultrathin alumina glasses that manifest as a BP. Measuring the dynamics of the $\mathrm{Al} / \mathrm{Al}_{2} \mathrm{O}_{3}$ is an important step towards understanding the atomistic degrees of freedom that affect the properties of alumina. One implication is that the low thermal conductivity of the $\mathrm{Al} / \mathrm{Al}_{2} \mathrm{O}_{3}$ interface is affected by the quasilocalized excitations in the oxide that lead to poor phonon transmission for THz-frequency excitations. It is also interesting to question whether the natural $\mathrm{THz}$-scale glass motions in the amorphous alumina have any effect on the two-level system noise at low temperature. At first glance the connection between the BP (which occurs in the THz/meV) energies and the TLSs (which occur at GHz frequencies) is not obvious. However, past theoretical studies showed that oxygen hopping modeled via the Schrödinger equation would lead to tunneling splittings in the $\mathrm{GHz}$ tunneling range for barrier heights of a few $\mathrm{meV}$ (i.e., at the same energy scale of the BP) to give rise to tunnel splittings in coarse agreement with experiment [26]. In the millikelvin regime, atomic motion over such a barrier is impossible except by quantum tunneling. However, at higher temperatures, it is likely that the underlying degree of freedom that contributes to the TLS is thermally populated and may contribute to the $\mathrm{THz}$ frequency signals in the classical regime. Furthermore, the possibility that the tunneling events are assisted or "dressed" by phonon excitations in the glass has also been proposed [3]. Future work needs to explore the relationship between the boson peak quasiphononic excitation with atomistic models of the two-level systems in amorphous alumina.

\section{ACKNOWLEDGMENTS}

J.H.C. and D.L.C. acknowledge the support of the Australian Research Council (ARC) via Grants No. DP140100375 and No. DE180100314. This work was partly supported by the ARC Centre for Excellence in Future Low-Energy Electronics (Grant No. CE170100039) and the Centre for Excellence in Exciton Science (Grant No. CE170100026). High performance computing was performed on the National Computational Infrastructure. This research used the JEOL JEM-ARM200F funded by an ARC LIEF grant (Grant No. LE120100104). Neutron spectroscopy was performed at the Australian Centre for Neutron Scattering (P7437).

\section{APPENDIX: MEASUREMENTS ON HEAT TREATED ALUMINA}

$\gamma-\mathrm{Al}_{2} \mathrm{O}_{3}$ is known to be hydrophillic in porous structures. Less is known about the affinity of water with the amor-

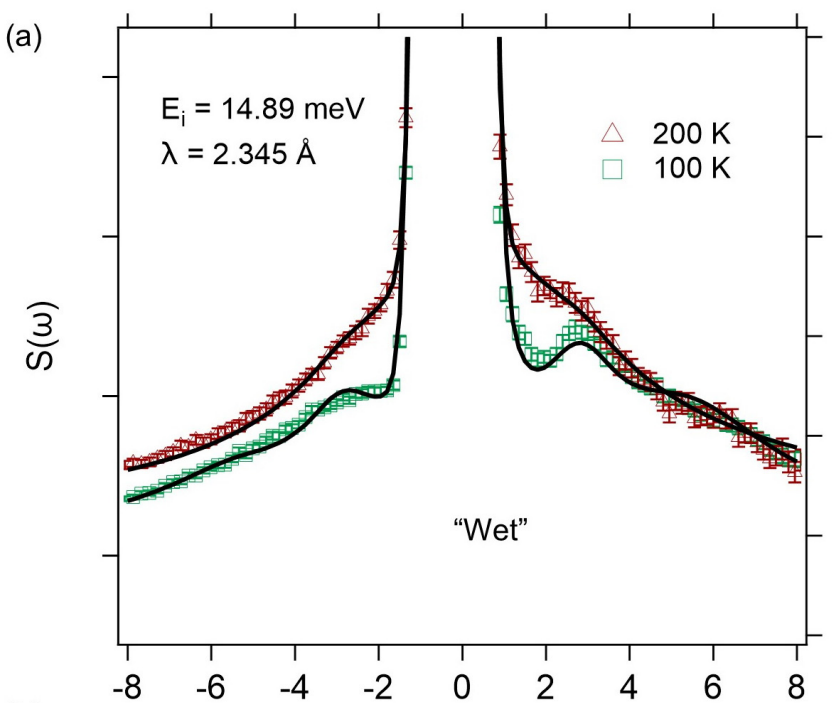

(b)

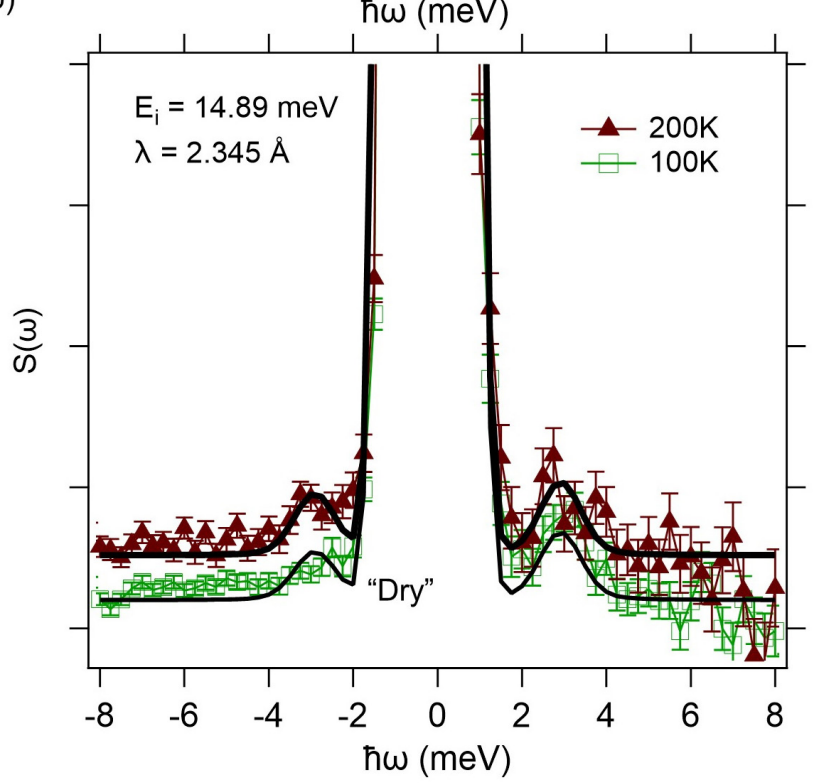

FIG. 6. Comparison of (a) as-exposed "wet" $\mathrm{Al}_{2} \mathrm{O}_{3}$ particles with (b) "dry" heat treated particles, where heating was performed in situ at $450 \mathrm{~K}$ in a vacuum environment.

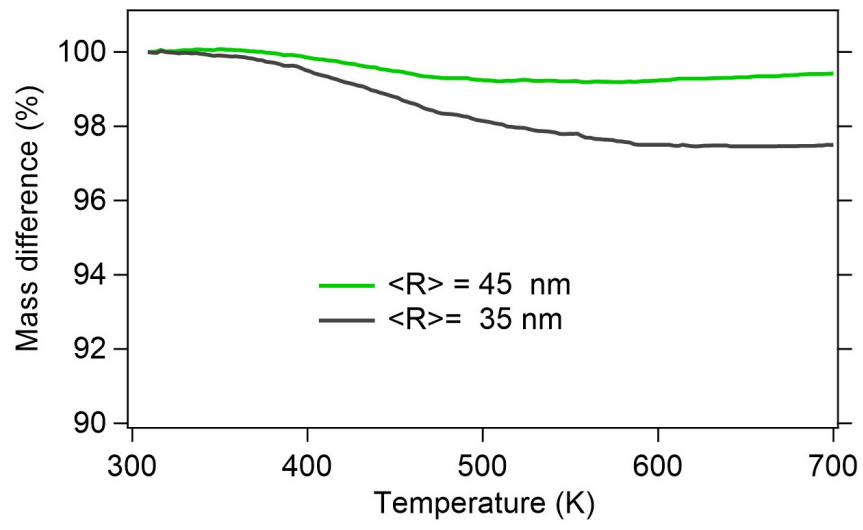

FIG. 7. Comparison of thermogravimetric analysis upon heating the $\langle R\rangle=45$ and $35 \mathrm{~nm}$ particles in a nitrogen atmosphere. The mass loss corresponds to the loss of bound water. 
phous alumina surface. As discussed above, the short-range structure in the amorphous phase shares certain structural motifs with the $\gamma$ phase. Thus, it is reasonable to expect amorphous alumina to exhibit chemical similarities with the $\gamma$ phase. The affinity for water on $\mathrm{AlO}_{x}$ requires investigation because hydrogen motion has been proposed to be a source of surface noise in quantum qubits based on amorphous alumina [51,52]. Neutron spectroscopy is extremely sensitive to small amounts of hydrogen because the total scattering cross-section of hydrogen is 1-2 orders of magnitude larger than the other elements. Our initial measurements on ambient exposed $\mathrm{Al}_{2} \mathrm{O}_{3}$ revealed a strong quasielastic component which is often the signature of hydrogen motion, including water and hydroxyls. Subsequent thermal gravimetric analysis measurements showed a small mass loss beginning at the boiling point of water. To investigate whether $\mathrm{H}_{2} \mathrm{O}$ was contributing to the quasielastic neutron signal, we carried out a preliminary in situ heating experiment as shown in
Fig. 6. After heating to $450 \mathrm{~K}$ and pumping under vacuum, the quasielastic signal is indeed reduced, however the BP feature remains observable. Complementary thermogravimetric analysis measurements are presented in Fig. 7 showing a mass loss at the boiling point of water. The smaller particles with $\langle R\rangle=35 \mathrm{~nm}$ have a larger mass loss than the particles with $\langle R\rangle=45 \mathrm{~nm}$. This may be expected if the water is surface bound because the smaller particles have a higher surface area $\left(\approx 30 \mathrm{~m}^{2} / \mathrm{g}\right)$. The broadness of the mass loss is suggestive of bound water (chemisorbed or physisorbed to a surface) because free water usually results in a sharp mass loss. Under the assumption that the water is surface bound, and has density similar to that of ordinary water, this mass loss corresponds to a coverage of approximately $1-2 \%$ of the alumina surface with $\mathrm{H}_{2} \mathrm{O}$, giving a density of approximately $0.3 \mathrm{H}_{2} \mathrm{O}$ per $\mathrm{nm}^{2}$. These measurements indicate that hydrogen in the form of water has an affinity for the surface of amorphous alumina.
[1] V. N. Novikov and A. P. Sokolov, Nature (London) 431, 961 (2004).

[2] K. Binder and W. Kob, Glassy Materials and Disordered Solids, revised ed. (World Scientific, Singapore, 2011).

[3] C. Müller, J. H. Cole, and J. Lisenfeld, Rep. Prog. Phys. 82, 124501 (2019).

[4] A. Loidl, Ann. Rev. Phys. Chem. 40, 29 (1989).

[5] M. F. Ando, O. Benzine, Z. Pan, J.-L. Garden, K. Wondraczek, S. Grimm, K. Schuster, and L. Wondraczek, Sci. Rep. 8, 5394 (2018).

[6] A. Hassan, L. Börjesson, and L. Torell, J. Non-Crystal. Sol. 172-174, 154 (1994).

[7] R. Zorn, M. Mayorova, D. Richter, A. Schönhals, L. Hartmann, F. Kremer, and B. Frick, in 5th International Workshop on Complex Systems, edited by M. Tokuyama, I. Oppenheim, and H. Nishiyama, AIP Conf. Proc. No. 982 (AIP, New York, 2008), p. 79 .

[8] R. Zorn, Phys. Rev. B 81, 054208 (2010).

[9] Z. Wang, K.-H. Liu, P. Le, M. Li, W.-S. Chiang, J. B. Leão, J. R. D. Copley, M. Tyagi, A. Podlesnyak, A. I. Kolesnikov, C.-Y. Mou, and S.-H. Chen, Phys. Rev. Lett. 112, 237802 (2014).

[10] F. Formisano and S. De Panfilis, Phys. Rev. Lett. 115, 149801 (2015).

[11] W. Steurer, A. Apfolter, M. Koch, W. E. Ernst, B. Holst, E. Søndergård, and J. R. Manson, Phys. Rev. Lett. 99, 035503 (2007).

[12] W. Steurer, A. Apfolter, M. Koch, W. E. Ernst, E. Søndergård, J. R. Manson, and B. Holst, Phys. Rev. Lett. 100, 135504 (2008).

[13] S. K. Lee, S. B. Lee, S. Y. Park, Y. S. Yi, and C. W. Ahn, Phys. Rev. Lett. 103, 095501 (2009).

[14] F. Reichel, L. P. H. Jeurgens, G. Richter, and E. J. Mittemeijer, J. Appl. Phys. 103, 093515 (2008).

[15] L. P. H. Jeurgens, W. G. Sloof, F. D. Tichelaar, and E. J. Mittemeijer, Phys. Rev. B 62, 4707 (2000).
[16] A. H. Tavakoli, P. S. Maram, S. J. Widgeon, J. Rufner, K. van Benthem, S. Ushakov, S. Sen, and A. Navrotsky, J. Phys. Chem. C 117, 17123 (2013).

[17] W.-C. Li, A.-H. Lu, W. Schmidt, and F. Schüth, Chemistry: A European Journal 11, 1658 (2005).

[18] M. J. Cyster, J. S. Smith, J. A. Vaitkus, N. Vogt, S. P. Russo, and J. H. Cole, Phys. Rev. Research 2, 013110 (2020).

[19] R. L. Fagaly, Rev. Sci. Inst. 77, 101101 (2006).

[20] J. Clarke and F. K. Wilhelm, Nature (London) 453, 1031 (2008).

[21] M. H. Devoret and R. J. Schoelkopf, Science 339, 1169 (2013).

[22] M. H. devoret and J. M. Martinis, Quantum Inf. Process. 3, 163 (2004).

[23] G. Wendin and V. S. Shumeiko, Low Temp. Phys. 33, 724 (2007).

[24] W. A. Phillips, Rep. Prog. Phys. 50, 1657 (1987).

[25] E. Paladino, Y. M. Galperin, G. Falci, and B. L. Altshuler, Rev. Mod. Phys. 86, 361 (2014).

[26] A. P. Paz, I. V. Lebedeva, I. V. Tokatly, and A. Rubio, Phys. Rev. B 90, 224202 (2014).

[27] D. Cortie, G. Casillas-Garcia, A. Squires, R. Mole, X. Wang, Y. Liu, Y.-H. Chen, and D. Yu, J. Phys. Condens. Mat. 31, 184003 (2019).

[28] L. Saviot, C. H. Netting, D. B. Murray, S. Rols, A. Mermet, A.-L. Papa, C. Pighini, D. Aymes, and N. Millot, Phys. Rev. B 78, 245426 (2008).

[29] D. Bozyigit, N. Yazdani, M. Yarema, O. Yarema, W. M. M. Lin, S. Volk, K. Vuttivorakulchai, M. Luisier, F. Juranyi, and V. Wood, Nature (London) 531, 618 (2016).

[30] T. Nakayama, Rep. Prog. Phys. 65, 1195 (2002).

[31] M. I. Lerner, E. A. Glazkova, A. S. Lozhkomoev, N. V. Svarovskaya, O. V. Bakina, A. V. Pervikov, and S. G. Psakhie, Powder Technology 295, 307 (2016).

[32] D. Yu, R. Mole, T. Noakes, S. Kennedy, and R. Robinson, J. Phys. Soc. Jap. 82, SA027 (2013).

[33] J. D. Gale, J. Chem. Soc., Faraday Trans. 93, 629 (1997).

[34] F. H. Streitz and J. W. Mintmire, Phys. Rev. B 50, 11996 (1994). 
[35] W. J. Mortier, S. K. Ghosh, and S. Shankar, J. Amer. Chem. Soc. 108, 4315 (1986).

[36] S. Ogata and T. J. Campbell, J. Phys.: Condens. Matter 10, 11449 (1998).

[37] G. Gutiérrez and B. Johansson, Phys. Rev. B 65, 104202 (2002).

[38] J. P. Sullivan, J. C. Barbour, R. G. Dunn, K. A. Son, L. P. Montes, N. Missert, and R. G. Copeland, in Proceedings of the Electrochemical Society Meeting, 1998 (unpublished).

[39] K. Hinsen, E. Pellegrini, S. Stachura, and G. R. Kneller, J. Comp. Chem. 33, 2043 (2012).

[40] J. Evertsson, F. Bertram, F. Zhang, L. Rullik, L. Merte, M. Shipilin, M. Soldemo, S. Ahmadi, N. Vinogradov, F. Carlà, J. Weissenrieder, M. Göthelid, J. Pan, A. Mikkelsen, J.-O. Nilsson, and E. Lundgren, Appl. Surf. Science 349, 826 (2015).

[41] P. Lamparter and R. Kniep, Physica B 234-236, 405 (1997).

[42] S. Davis and G. Gutiérrez, J. Phys. Condens. Matt. 23, 495401 (2011).

[43] T. Chatterji, Y. Su, G. N. Iles, Y.-C. Lee, A. P. Khandhar, and K. M. Krishnan, J. Magn. Magn. Mat. 322, 3333 (2010).
[44] B. Fultz, C. C. Ahn, E. E. Alp, W. Sturhahn, and T. S. Toellner, Phys. Rev. Lett. 79, 937 (1997).

[45] A. F. Yue, A. B. Papandrew, O. Delaire, B. Fultz, Z. Chowdhuri, R. M. Dimeo, and D. A. Neumann, Phys. Rev. Lett. 93, 205501 (2004).

[46] W. Y. Ching, L. Ouyang, P. Rulis, and H. Yao, Phys. Rev. B 78, 014106 (2008).

[47] R. Heid, D. Strauch, and K.-P. Bohnen, Phys. Rev. B 61, 8625 (2000).

[48] M. T. Dove, M. J. Harris, A. C. Hannon, J. M. Parker, I. P. Swainson, and M. Gambhir, Phys. Rev. Lett. 78, 1070 (1997).

[49] S. P. Bayrakci, D. A. Tennant, P. Leininger, T. Keller, M. C. R. Gibson, S. D. Wilson, R. J. Birgeneau, and B. Keimer, Phys. Rev. Lett. 111, 017204 (2013).

[50] C. S. Gorham, J. T. Gaskins, G. N. Parsons, M. D. Losego, and P. E. Hopkins, Appl. Phys. Lett. 104, 253107 (2014).

[51] A. M. Holder, K. D. Osborn, C. J. Lobb, and C. B. Musgrave, Phys. Rev. Lett. 111, 065901 (2013).

[52] L. Gordon, H. Abu-Farsakh, A. Janotti, and C. Van de Walle, Sci. Rep. 4, 7590 (2015). 